\title{
Efficacy and Safety of Sonidegib in Adult Patients with Nevoid Basal Cell Carcinoma Syndrome (Gorlin Syndrome): Results from a Phase 2, Double-Blind, Randomized Trial
}

This article was published in the following Dove Press journal:

Clinical, Cosmetic and Investigational Dermatology

John T Lear'

Axel Hauschild (DiD ${ }^{2}$

Eggert Stockfleth ${ }^{3}$

Nicholas Squittieri ${ }^{4}$

Nicole Basset-Seguin (1D ${ }^{5}$

Reinhard Dummer $\mathbb{D D}^{6}$

'Manchester Royal Infirmary, Manchester, UK; ${ }^{2}$ Klinik Für Dermatologie, Venerologie Und Allergologie

Universitätsklinikum Schleswig-Holstein, Kiel, Germany; ${ }^{3}$ Universitätshautklinik

Bochum, Bochum, Germany; ${ }^{4}$ Sun Pharmaceutical Industries, Inc., Princeton, NJ, USA; ${ }^{5}$ Department of Dermatology, Hôpital Saint Louis, Paris, France; ${ }^{6}$ Skin

Cancer Center University Hospital,

Zürich, Switzerland
Correspondence: John T Lear University of Manchester, 46 Grafton Street, Manchester MI3 9NT, UK

Tel +44 I6I 2764173

$\mathrm{Fax}+44$ I6I 276888 I

Email john.lear@srft.nhs.uk

\section{Introduction}

Nevoid basal cell carcinoma syndrome (NBCCS), or Gorlin syndrome, is a rare hereditary disease characterized by the development of multiple cutaneous basal cell carcinomas (BCCs) from a young age. ${ }^{1}$ Loss-of-function germline mutations in the hedgehog-related patched 1 (PTCH1) tumor suppressor gene are the most common cause of NBCCS. ${ }^{1}$ The hedgehog signaling pathway plays a major role in embryonic development, and in adulthood, is involved in the renewal and maintenance of distinct tissues, including hair follicles, muscle stem cells, and gastric epithelium. ${ }^{2}$ Its abnormal activation is thought to drive the formation of both sporadic BCCs and those resulting from NBCCS. ${ }^{1}$ Patients with NBCCS inherit one inactive copy of PTCH1 and then acquire a "second-hit" mutation, resulting in hedgehog pathway activation and BCC formation. ${ }^{1}$ Mutations in Suppressor of fused (SUFU) or the PTCH1 homolog PTCH2 have also been found in a subset of patients meeting criteria for NBCCS. ${ }^{1,3}$

Treatment of BCCs in patients with NBCCS can be extremely difficult due to the large number of tumors. Patients with a limited number of BCCs may benefit from surgical excision. ${ }^{3}$ However, patients with aggressive or recurrent tumors, as well as tumors in delicate or high-risk areas, may benefit from Mohs micrographic surgery, which is lengthy and costly. ${ }^{3}$ Multiple surgical procedures can be a source of discomfort, pain, and disfigurement for patients with NBCCS, leading to an unmet need for nonsurgical and minimally invasive treatment options.

Sonidegib (Odomzo ${ }^{\circledR}$; Sun Pharmaceutical Industries, Inc.; Cranbury, NJ), a hedgehog pathway inhibitor (HPI), is approved for the treatment of adult patients with locally advanced BCC (laBCC) that has recurred following surgery or radiation therapy, or those who are not candidates for surgery or radiation therapy. ${ }^{4}$ Approval was based on results from the BOLT (BCC outcomes with LDE225 [sonidegib] treatment) study. ${ }^{5,6}$ Here we report the results of an exploratory study evaluating the safety, preliminary efficacy, and pharmacokinetics of sonidegib in patients with NBCCS after 12 weeks of treatment. 


\section{Methods}

This phase 2, double-blind, randomized study (NCT0 1350115) adhered to the Declaration of Helsinki and International Council for Harmonisation Consolidated Guideline E6 for Good Clinical Practice. Approval to the study protocol and all amendments was provided by the Ethics Committees at Erasmus Hospital, UZ Leuven, the Christian-Albrechts-University of Kiel, and the University of Vienna; and by the Institutional Review Board at Aurora, Ontario. All patients provided written informed consent before enrollment.

Adults with at least two BCCs were eligible to enroll if they met at least one of the following diagnostic criteria for NBCCS: keratocystic odontogenic tumors, either from medical history or optional radiography at screening; at least three palmar and/or plantar pits; history of bilamellar calcification of the falx cerebri or bifid, fused, or markedly splayed ribs; first-degree relatives with NBCCS; and PTCH1 mutation identified in genome from nontumor tissue. Patients with a histologically confirmed diagnosis of laBCC or metastatic BCC (mBCC) not amenable to radiation therapy or curative surgery were excluded from the study, as were patients with an ongoing or recent history of severe, progressive, or uncontrolled systemic disease. Additional key exclusion criteria included use of topical treatments for BCCs in the 4 weeks prior to the first dose of study drug; any prior use of HPI; and use of photodynamic therapy, radiation, or systemic treatments known to affect BCCs.

Randomization was planned 6:1 to sonidegib $400 \mathrm{mg}$ daily or placebo for 12 weeks. At screening, one target BCC was selected per patient; the target $\mathrm{BCC}$ was a nonrecurring, previously untreated or unbiopsied BCC 5-20 mm long in a location amenable to surgical excision. Clinical clearance of the target BCC was assessed every 2 weeks until the lesion was excised at week 16. After week 19, patients returned approximately every 2 months for the next 6 months to assess tumor burden and safety.

The primary endpoint was target $\mathrm{BCC}$ clinical clearance at week 16 , with demonstrated $\geq 60 \%$ posterior probability that $\geq 50 \%$ of the patients receiving sonidegib exhibited complete clinical clearance. Clinical clearance was assessed by a blinded, experienced, and qualified dermatologist who compared the presentation of the target BCC during the study visit to a macroscopic image of the $\mathrm{BCC}$ at baseline. For consistency, the same investigator assessed a particular BCC at every study visit. The response was evaluated on a 6-grade scale from "worsening" to "complete clearance" (all scale grades summarized in Table 1), where complete clearance was defined as the absence of any clinical signs of carcinoma, except postinflammatory changes such as erythema, changes in pigmentation, or scarring. This approach was designed to be simple and reproducible and to replicate evaluation of a BCC in clinical practice.

Secondary assessments included target BCC histological clearance at week 16 and tumor burden assessed by BCC counts every 2-4 weeks and at follow-up visits. Safety

Table I Efficacy and Safety Outcomes

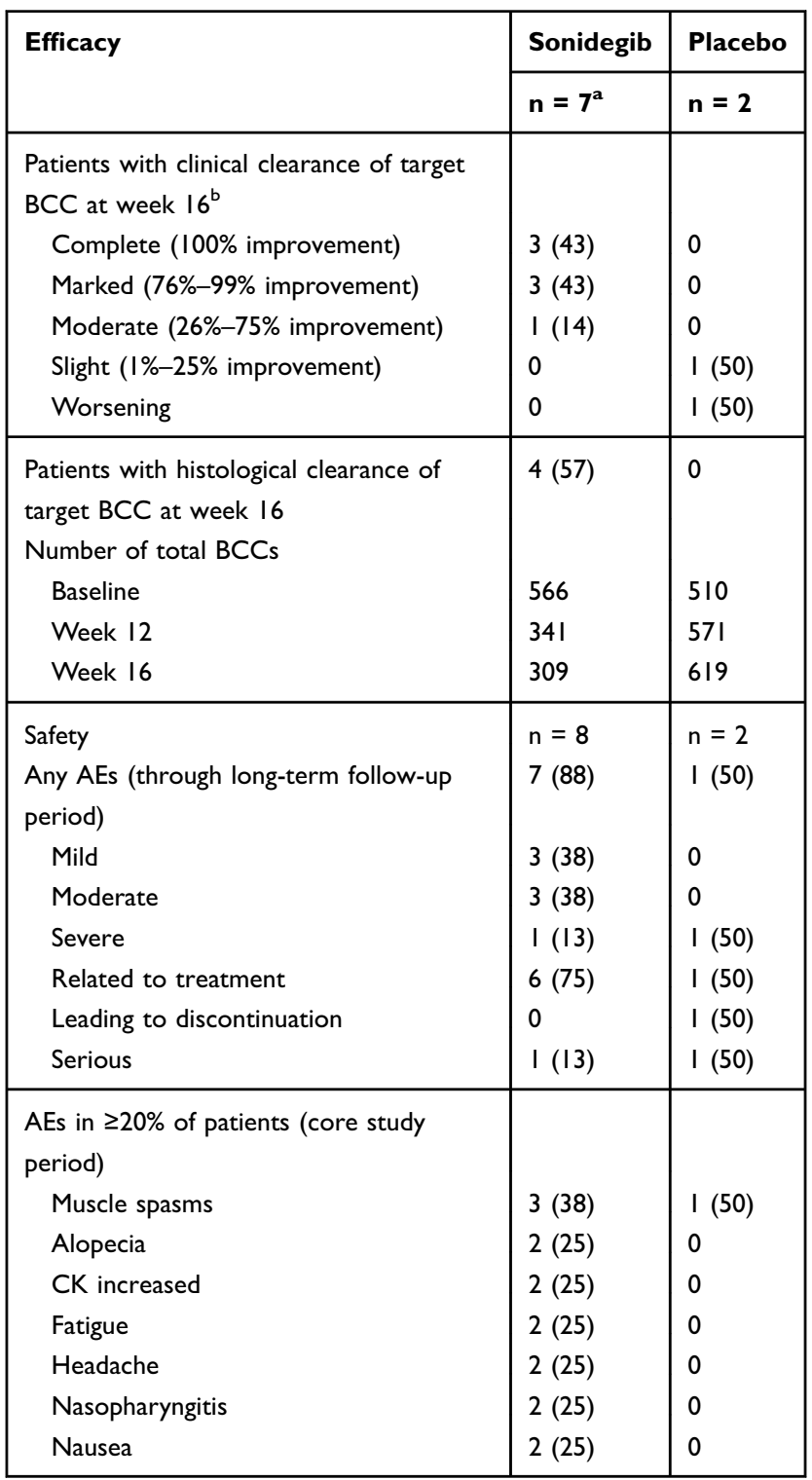

Notes: Data presented as $\mathrm{n}(\%)$ of total patients in treatment arm unless otherwise indicated. ${ }^{a}$ One patient was excluded from efficacy analysis due to receipt of placebo in 5 of 13 doses. ${ }^{b}$ One target BCC was counted per patient and assigned to I of 5 listed clearance categories.

Abbreviations: AE, adverse event; $\mathrm{BCC}$, basal cell carcinoma; $\mathrm{CK}$, creatine kinase. 
assessments included adverse event (AE) monitoring through the study and for up to 6 months following core study completion. AE toxicity was graded according to the National Cancer Institute common terminology criteria for AEs version 4.0.

\section{Results}

Ten patients were enrolled; 8 and 7 completed the core study and long-term follow-up, respectively (Figure 1). Due to AEs and laboratory assessment abnormalities, 2 patients discontinued sonidegib treatment on days 65 and 72 , respectively. These patients were still included in the analysis and enrolled in the long-term follow-up, because they had completed a significant part of the 12-week treatment period. Median (range) age was 53 (37-68) years and (range) age was (64-68) years for sonidegib vs placebo, respectively. Male patients comprised 50\% and $100 \%$ of the sonidegib and placebo groups, respectively.

Complete clinical clearance at week 16 was observed in 3 of 7 (43\%) patients receiving sonidegib (Table 1). One patient receiving sonidegib was excluded from analysis due to a protocol deviation. In the placebo group, $1(50 \%)$ patient exhibited slight clearance at week 16 and 1 (50\%) exhibited worsening. A 35\% posterior probability of the true complete clinical clearance rate being $\geq 50 \%$ was derived from the $43 \%$ observed complete clinical clearance. Since posterior probability was $<60 \%$, the primary endpoint was not met. At week 16, 4 (57\%) patients receiving sonidegib demonstrated target BCC complete histological clearance vs 0 receiving placebo.

At baseline, the total BCC number in the sonidegib group was 566 vs 510 for the placebo group (Table 1). At weeks 12 and 16, the total BCC for the sonidegib group was 341 and 309, respectively, vs 571 and 619 for the placebo group. The number of BCCs per patient was highly variable; the 4 patients with the highest number of BCCs in the sonidegib group at baseline had between 65 and 214 tumors, while several patients had $<5$ tumors.

Most AEs were mild-to-moderate (Table 1). One patient receiving sonidegib experienced a serious $\mathrm{AE}$ (face cellulitis) during the long-term follow-up that was not suspected to be related to sonidegib. One patient receiving placebo experienced two serious AEs; worsening of $\mathrm{BCC}$ on the head and residual $\mathrm{BCC}$ growth after recent surgery. No deaths occurred. The most common AE was

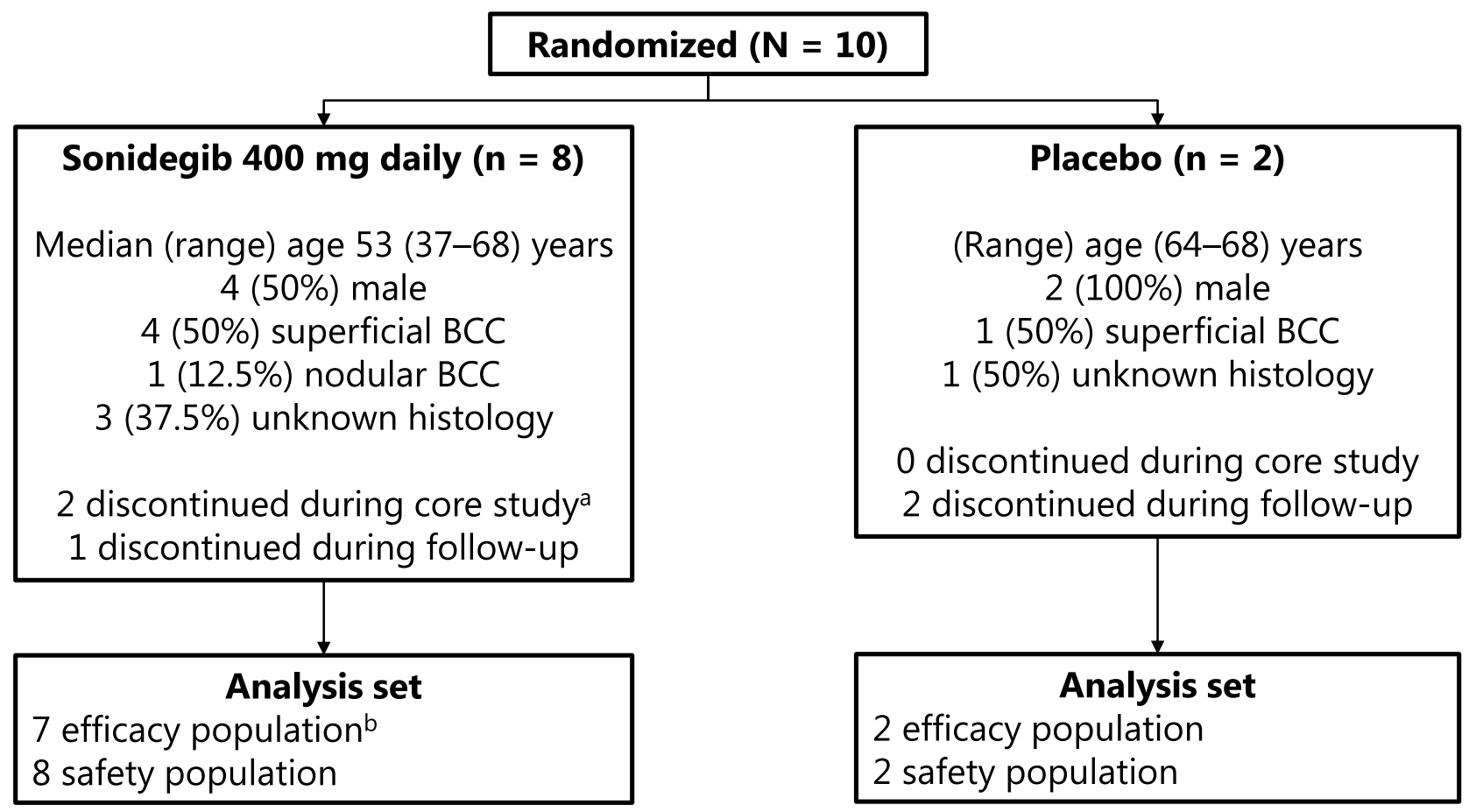

Figure I Patient disposition.

Notes: ${ }^{a}$ Patients were still included in the analysis and enrolled in long-term follow-up due to completion of most of the treatment period. ${ }^{b}$ One patient was excluded from efficacy analysis due to receipt of placebo in 5 of 13 doses.

Abbreviation: BCC, basal cell carcinoma. 
muscle spasms, reported in $3(38 \%)$ and 1 (50\%) patients receiving sonidegib and placebo, respectively. Alopecia and elevated creatine kinase were each reported in 2 (25\%) and 0 patients receiving sonidegib and placebo, respectively.

\section{Discussion}

While the primary efficacy endpoint in this study was not met, potentially due to small sample size, oral administration of sonidegib $400 \mathrm{mg}$ daily for 12 weeks resulted in complete clinical clearance of a target $\mathrm{BCC}$ in 3 of 7 patients with NBCCS. The remaining four patients experienced at least moderate clinical clearance. Overall, all patients receiving sonidegib $400 \mathrm{mg}$ experienced some clearance of $\mathrm{BCC}$, and no patient receiving sonidegib had worsening of the target BCC. There was a discrepancy between clinical and histological BCC clearance, potentially due to remaining erythema or inflammation confounding clinical evaluation of BCCs.

The efficacy and safety of sonidegib were previously evaluated in patients with laBCC or $\mathrm{MBCC}$ in the BOLT study. ${ }^{5,6}$ The approved sonidegib dose of $200 \mathrm{mg}$ daily achieved an objective response rate (ORR) by central review (95\% confidence interval [CI]) of $43 \%(28 \%-59 \%)$ and $15 \%(2 \%-45 \%)$ in patients with laBCC and $\mathrm{mBCC}$, respectively, after up to 6 months of treatment. ${ }^{5}$ In followup results at 42 months, sonidegib $200 \mathrm{mg}$ daily maintained its efficacy profile with ORR per central review $(95 \% \mathrm{CI})$ of $56.1 \%(43.3 \%-68.3 \%)$ and $7.7 \%(0.2 \%-36.0 \%)$ in laBCC and $\mathrm{mBCC}$, respectively. ${ }^{6}$ The most common AEs at 42 months in patients (n [\%]) receiving sonidegib $200 \mathrm{mg}$ daily included muscle spasms (43 [54.4\%]), alopecia (39 [49.4\%]), and dysgeusia $(35[44.3 \%]){ }^{6}$ Guidelines were established for the management of AEs using dose interruptions. $^{5}$

Vismodegib (Erivedge ${ }^{\circledR}$, Genentech, San Francisco, CA), a HPI, was evaluated at a dose of $150 \mathrm{mg}$ daily vs placebo in a randomized, double-blind, phase 2 study in patients with NBCCS. ${ }^{7}$ Treatment with vismodegib led to a significantly reduced mean rate of appearance of surgically eligible BCCs vs placebo (2 vs 34 new BCCs per patient per year, respectively, $P<0.0001)$. After discontinuation of vismodegib, the rate of appearance of new surgically eligible BCCs was not significantly different from the placebo group ( 0.06 vs 0.12 new BCCs per month for vismodegib vs placebo, respectively, $P=$ 0.06). AEs in the vismodegib group-including alopecia $(100 \%)$, muscle cramps (100\%), and dysgeusia (93\%)- limited the use of vismodegib and led to discontinuation in 19 of 25 patients at 1 study site $(76 \%){ }^{7}$ The prevalence of these AEs in the current study was lower when compared with BOLT and the study of vismodegib in NBCCS, potentially due to shorter treatment duration or smaller patient numbers.

Limitations of the current study include the small sample size, with only two patients in the placebo arm, which contributed to limited statistical evaluation of efficacy and safety results. In $37.5 \%$ of patients receiving sonidegib, tumor histology was unknown. In addition, efficacy outcomes were assessed at 16 weeks of treatment, which may have been too short to fully assess the clinical effects of sonidegib in patients with NBCCS. Reporting of standard oncological measures, such as objective response rate and best overall response, would contribute to a more complete assessment of sonidegib efficacy in NBCCS; however, the small sample size, short duration, and exploratory design of the study may limit the conclusions that can be drawn from these measures.

This is the first report demonstrating the efficacy and safety of sonidegib in NBCCS. Sonidegib showed promising efficacy as demonstrated by partial or complete clinical clearance of target BCCs and decreased tumor burden in all patients, and histological clearance in $57 \%$ of the patients. Overall, sonidegib appeared well tolerated. Further studies are needed to thoroughly evaluate the sonidegib safety and efficacy trends observed in patients with NBCCS.

\section{Data Sharing Statement}

Data and other documents will be made available after publication, with no end date, to anyone who submits a reasonable request to the study sponsor.

\section{Acknowledgments}

This study was sponsored and funded by Novartis. Writing and editorial support for manuscript preparation were provided by Ginny Feltzin, PhD, of AlphaBioCom, LLC, and funded by Sun Pharmaceutical Industries, Inc., Princeton, NJ, USA. All authors met the International Council of Medical Journal Editors criteria and received neither honoraria nor payment for authorship.

\section{Disclosure}

JTL receives personal fees from Novartis and Sun Pharmaceutical Industries, Inc. RD receives grants and personal fees from Bristol Myers-Squibb, GlaxoSmithKline, Merck 
Sharpe and Dohme, Novartis, and Roche. AH serves as consultant to Roche, Novartis, Amgen, Celgene, GlaxoSmithKline, MedImmune, MelaSciences, Merck Serono, Oncosec, and Eisai; and received speaker fees, travel grants, and research funding from Roche, Novartis, Amgen, Celgene, GlaxoSmithKline, MedImmune, MelaSciences, Merck Serono, Oncosec, and Eisai. NS and NBS are employees of Sun Pharmaceutical Industries, Inc.; NBS receives honoraria from Galderma, Leo, Pierre Fabre, Novartis, and Roche; consulting fees from Galderma, Leo, Pierre Fabre, Novartis, and Roche; patents, royalties, or other intellectual property from Genentech/F. Hoffmann-La Roche, Ltd.; and travel, accommodations, or expenses from Galderma, Leo, and Roche. ES has served as a consultant to LEO Pharma $\mathrm{A} / \mathrm{S}$. RD receives grants and personal fees from Amgen, Bristol Myers-Squibb, Catalym, GlaxoSmithKline, Merck Sharpe and Dohme, Novartis, Roche, Pierre Fabre, Sun Pharmaceutical Industries, Inc, Sanofi, Second Genome, and Takeda. The authors report no other conflicts of interest in this work.

\section{References}

1. Bresler SC, Padwa BL, Granter SR. Nevoid basal cell carcinoma syndrome (gorlin syndrome). Head Neck Pathol. 2016;10 (2):119-124. doi:10.1007/s12105-016-0706-9

2. Petrova R, Joyner AL. Roles for Hedgehog signaling in adult organ homeostasis and repair. Development. 2014;141(18):3445-3457. doi: $10.1242 /$ dev. 083691

3. Kiwilsza M, Sporniak-Tutak K. Gorlin-Goltz syndrome-a medical condition requiring a multidisciplinary approach. Med Sci Monit. 2012;18(9):Ra145-153. doi:10.12659/MSM.883341

4. Odomzo (sonidegib capsules). Full Prescribing Information. Cranbury, NJ, USA: Sun Pharmaceutical Industries, Inc; 2017.

5. Migden MR, Guminski A, Gutzmer R, et al. Treatment with two different doses of sonidegib in patients with locally advanced or metastatic basal cell carcinoma (BOLT): a multicentre, randomised, double-blind phase 2 trial. Lancet Oncol. 2015;16(6):716-728. doi:10.1016/S1470-2045(15)70100-2

6. Dummer R, Guminksi A, Gutzmer R, et al. Long-term efficacy and safety of sonidegib in patients with advanced basal cell carcinoma: 42-month analysis of the phase 2 randomised, double-blind BOLT study. Br J Dermatol. 2019. doi:10.1111/bjd.18552

7. Tang JY, Ally MS, Chanana AM, et al. Inhibition of the hedgehog pathway in patients with basal-cell nevus syndrome: final results from the multicentre, randomised, double-blind, placebo-controlled, phase 2 trial. Lancet Oncol. 2016;17(12):1720-1731. doi:10.1016/S14702045(16)30566-6

\section{Publish your work in this journal}

Clinical, Cosmetic and Investigational Dermatology is an international, peer-reviewed, open access, online journal that focuses on the latest clinical and experimental research in all aspects of skin disease and cosmetic interventions. This journal is indexed on CAS.
The manuscript management system is completely online and includes a very quick and fair peer-review system, which is all easy to use. Visit http://www.dovepress.com/testimonials.php to read real quotes from published authors. 